\title{
Comparison of temperature measurements in bladder, rectum and pulmonary artery in patients after cardiac surgery
}

\author{
Hendrika Wollerich, Farouq Ismael, Maarten W. Nijsten, Willem Dieperink \\ Department of Critical Care, University of Groningen, University Medical Center Groningen, Groningen, Netherlands \\ Email: w.dieperink@umcg.nl
}

Received 10 September 2012; revised 12 October 2012; accepted 25 October 2012

\begin{abstract}
In many patients in the intensive care unit (ICU) continuous temperature monitoring is performed with rectal probes. Currently there are more options to measure temperature in critically ill patients. Since bladder catheters are routinely used in the majority of ICU patients, using bladder temperature (Tb) could do away with rectal probes. In this prospective study, we compared $\mathrm{Tb}$, rectal temperature $(\mathrm{Tr})$ and pulmonary artery catheter temperature (Tpa) in patients who underwent cardiac surgery. We also evaluated if urinary production affected Tb. Patients admitted after cardiac surgery with sensors in place for measurement of $\mathrm{Tr}, \mathrm{Tb}$ and $\mathrm{Tpa}$ upon arrival at the ICU were included. Diuresis was recorded every hour. Data were collected until $24 \mathrm{~h}$ after admission or until ICU discharge. Nineteen consecutive patients ( 9 males; mean age 61 years) were evaluated. Over a median observation period of 17 hours $382 \mathrm{~Tb}, 333 \mathrm{Tr}$ and 371 Tpa measurements were recorded. Linear correlations $(R)$ between $T b$ and $T r$, between $T b$ and $T p a$ and between $\mathrm{Tr}$ and Tpa were $0.95,0.95$ and 0.91 respectively $(\mathrm{P}<0.001)$. Bland-Altman analysis demonstrated no relation between temperature and the (TbTr) offset. No relation of diuresis with (Tb-Tr) or (Tb-Tpa) was observed. After cardiac surgery, bladder temperature performed as well as conventional rectal probes with no interference of diuresis on bladder temperature measurement. Thus, the use of bladder temperature probes may be preferable to rectal probes in patients after cardiac surgery.
\end{abstract}

Keywords: Intensive Care; Cardiac Surgery; Body Temperature

\section{INTRODUCTION}

Accurate and precise measurement of body temperature is important for in the timely detection of fever or hypo- thermia in patients in the intensive care unit (ICU). No uniform practice regarding temperature measurement exists. A variety of devices are used to measure body temperature at diverse anatomical locations in two possible temperature scales. Traditionally the temperature measured within the pulmonary artery (Tpa) with a the SwanGanz catheter (PAC), that is typically only used in patients with complex hemodynamic problems, is considered the "gold standard" for the measurement of body temperature [1].

Nevertheless, for routine clinical use in ICU patients, using a PAC is clearly inapplicable.

In many ICU's continuous rectal temperature measurement (Tr) is standard practice [2]. Placement of thermometers in the rectum is more invasive than in the axilla or groin. A rectal probe can be distressing as well as embarrassing for patients. Moreover, for inserting the probe, patients must be placed on their side, a maneuver that often requires two nurses to safely turn the patient. The rectal temperature probe can also result in mucosal injury, or even perforation of the rectum [3]. Tr can also be affected by the passage of stools and the probe can dislocate easily. On the other hand $\mathrm{Tr}$ is more accurate than less invasive measurement methods such as at the axilla or inguinal [4].

Urinary bladder temperature (Tb), measurement of temperature with a specially designed temperature probe embedded in a Foley urinary catheter, could be a favorable alternative to $\mathrm{Tr}$ in the ICU because rectal probe insertion is not required without the need for additional procedures $[5,6]$. In several patient groups it has been established that $\mathrm{Tb}$ generally corresponds well to the Tpa, the "gold standard" $[7,8]$. It has been suggested, that the accuracy and precision of $\mathrm{Tb}$ might be influenced by the urinary flow rate [5,9]. In 22 ICU patients in which temperature was simultaneously monitored with PAC it has been demonstrated that the reliability of $\mathrm{Tb}$ is higher than either $\operatorname{Tr}$ or inguinal or axillary temperature measurements $[6,10]$. 
It is not known if the same holds true for the reliability of $\mathrm{Tb}$ during the direct post-operative phase in patients admitted after cardiac surgery. Cardiac surgery involves the opening of the chest and frequently the use of a heart-lung machine. Thus during and after such surgery important swings in hemodynamics and temperature can occur. This may be particularly relevant given the large thermal gradients from core to periphery that occur during anesthesia and cardiac surgery [5].

\section{Objective}

In a prospective study we tested the hypothesis that after cardiac surgery $\mathrm{Tb}$ adequately reflects $\mathrm{Tpa}$, and at least as good as Tr. We also examined the effect of varying urinary flow rate on the $\mathrm{Tb}$.

\section{METHODS}

During a 10-month study period (June 2009 to March 2010) consecutive patients aged $>18$ years, who were undergoing cardiac surgery and admitted to the department of critical care were evaluated for this study. Patients, with a ileal conduit urinary diversion also known as Bricker ileal conduit, or patients with suprapubic catheters, or other patients where urethral catheterization was contraindicated as well as patients who were undergoing renal replacement therapy were excluded.

Only patients with both a PAC, a urinary bladder catheter with temperature probe $(\mathrm{Tb})$ and a rectal temperature probe $(\mathrm{Tr})$ were included.

$\mathrm{Tb}$ was measured by a probe incorporated in a Foley urinary catheter (DeRoyal, Powell, USA). Tpa was measured by a pulmonary artery catheter (PAC) (Edwards, Irvine California). Tr was measured by a digital thermometer probe (Esophageal/Rectal temperature probe $25^{\circ} \mathrm{C}-45^{\circ} \mathrm{C}$, Philips Medical Systems, Andover, USA) designed for axillar and rectal use. The temperature sensors and the PAC were respectively connected to a temperature and cardiac output module of the bedside monitor (MP70, Philips Medical Systems, Eindhoven, the Netherlands). Temperature values were recorded in degrees Celsius every 30 minutes by the ICU nurses. The urinary production in $\mathrm{ml}$ was recorded every hour. All data were collected until $24 \mathrm{~h}$ after admission or until ICU discharge if this took place within $24 \mathrm{~h}$.

Tpa was considered the reference value. Thus $\mathrm{Tb}$ was compared with Tpa, and Tr was compared with Tpa using regression and Bland and Altman analysis.

Since no additional interventions were performed informed consent was not required for this anonymized analysis of data. The institutional review board of the University Medical Center Groningen approved this observational study (METc 2008/279).

\section{RESULTS}

During the study period, 19 consecutive patients admitted to the department of critical care after cardiac surgery were included. For 9 men and 10 women with a mean age of $61 \pm 13$ (SD) years, we recorded temperatures over a median observation period of 17 hours. A total of $382 \mathrm{~Tb}, 333 \mathrm{Tr}$ and $371 \mathrm{Tpa}$ measurements were analyzed (Table 1). We also recorded 291 diuresis measurements.

Correlation coefficients between $\mathrm{Tb}$ and $\mathrm{Tr}$, between $\mathrm{Tb}$ and Tpa (Figure 1) and between $\mathrm{Tr}$ and Tpa were $0.95,0.95$ and 0.91 respectively $\left(\mathrm{P}<10^{-9}\right)$. The mean offsets for expressed as (Tb-Tr), (Tb-Tpa) and (Tr-Tpa) were $-0.05^{\circ} \mathrm{C},+0.05^{\circ} \mathrm{C}$ and $+0.10^{\circ} \mathrm{C}$ respectively, indicating that $\operatorname{Tr}$ was the least reliable of the three measurements.

Bland-Altman analysis demonstrated no relation between temperature and the ( $\mathrm{Tb}-\mathrm{Tr}$ ) offset (Figure 2). No relation of diuresis with (Tb-Tr) or (Tb-Tpa) was observed. The two outlying values visible in Figures 1 and 2 concerned immediate post-operative patients with very cold peripheries and consequently high temperature gradients. For both these outliers Tr deviated more from Tpa than $\mathrm{Tb}$.

\section{DISCUSSION}

Measuring body temperature in critically ill patients is a part of routine nursing care. However, it is still unclear which method and device is ideal for measuring accurate, reliable affordable, comfortable and safe body temperature. Nurses therefore must have the knowledge and skills related to the various temperature measuring

Table 1. Patient characteristics.

\begin{tabular}{lc}
\hline Number of patients & 19 \\
Age, mean, SD, (range) & $61.1 \pm 13.4(32-83)$ \\
Gender, male/female & $9 / 10$ \\
Admission diagnoses: & $9(47.3)$ \\
$\quad$ Valve replacement, n (\%) & $5(26.3)$ \\
$\quad$ CABG, n (\%) & $5(30.3)$ \\
$\quad$ Cardiovascular other, n (\%) & $171.1 \pm 8.2(156-189)$ \\
Height (cm), mean, SD, (range) & $79.0 \pm 16.2(51-105)$ \\
Weight (kg), mean, SD, (range) & $1.9 \pm 0.2(1.53-2.37)$ \\
BSA (m ${ }^{2}$ ), mean, SD, (range) & $44.8 \pm 15.7$ \\
APACHE IV score, mean, \pm SD, (range) & $30.5 \pm 8.1$ \\
SAPS II score, mean, \pm SD & $88 \pm 79(5-400)$ \\
\hline Diuresis, ml/hr, mean, \pm SD, (range) &
\end{tabular}

$\mathrm{SD}=$ Standard Deviation, $\mathrm{CABG}=$ Coronary Artery Bypass Graft Surgery, BSA $=$ Body Surface Area, APACHE II = Acute Physiologic and Chronic Health Evaluation, SAPS II = Simplified Acute Physiology Score. 


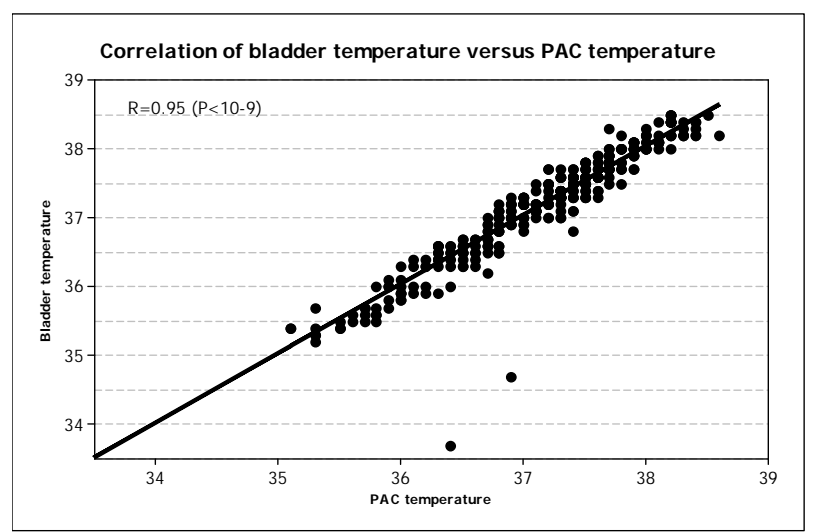

Figure 1. Correlation plot of $\mathrm{Tb}$ versus Tpa showing high correlation between the two temperature measuring methods.

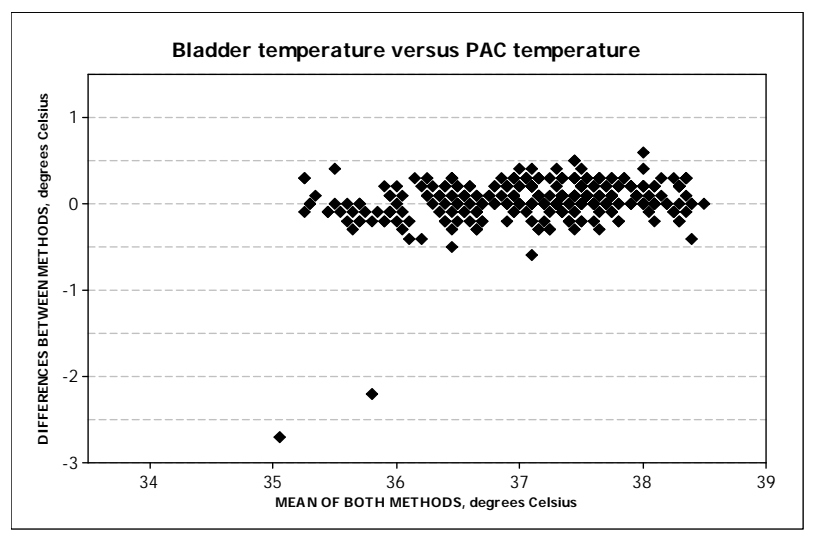

Figure 2. Bland and Altman Plot of the data obtained from paired samples of bladder and PAC temperature. Correlation $\mathrm{R}$ $=0.95\left(\mathrm{P}<10^{-9}\right)$.

methods and associated devices to make good clinical decisions. In the ICU, Tb measurement has gained increased acceptance and is simplified by the fact that most patients have an indwelling Foley catheter.

In many clinical studies Tpa is considered as the most optimal representation of internal body temperature [8, $11,12]$. However, for temperature measurement only, Tpa is obviously not a practical technique as the invasive pulmonary artery catheter is only required in a minority of ICU patients. Inserting rectal thermometers is usually uncomplicated, although it can occasionally cause mucosal injury. However, these probes can be easily dislocated by stool movements or by the application of suppositories and they are definitely associated with additional patient discomfort. At our ICU most patients have bladder catheters, making the use of a catheter with an incorporated temperature probe therefore not additionally invasive. Thus $\mathrm{Tb}$ could be the preferred temperature device in ICU patients.

The data in our study agree with those found in other patient groups where neither $\mathrm{Tb}$ nor $\mathrm{Tr}$ exactly correlate with Tpa [13-17]. However, bladder temperature gener- ally did as well or better compared to Tr.

In spite of the fact that devices for $\mathrm{Tb}$ measurement are more expensive than $\operatorname{Tr}$ devices, the overall costs for $\mathrm{Tb}$ are lower because $\mathrm{Tr}$ probes must be changed more often than $\mathrm{Tb}$ probes. Thus, although overall costs may vary according to local circumstances, in our setting the use of $\mathrm{Tb}$ is associated with reduced yearly cost decrease of more than $€ 4000$ for our unit.

There are some limitations of the present study. First, none of the CE-certified thermometers were tested or compared with certified laboratory thermometers. On the other hand, all equipment and materials in our hospital are routinely monitored and checked regularly, and Tpa is regarded as the gold standard.

Second, rectal temperature measurement could be affected by the depth of inserting the probe, conditions affecting local blood flow and the presence of stools. These factors were not assessed in the our study since we assumed that interference of stools or probe dislocation would be less important, so early after insertion and surgery. But if these factors come into play later on, we expect them to work particularly to the disadvantage of the rectal temperature measurement, not the bladder measurement.

And finally, we had a small number of measurements in patients who were severely hypothermic or hyperthermic. Hence, further studies with larger sample sizes including hypo and hyperthermic patients might further strengthen the results of this study.

\section{CONCLUSIONS}

In conclusion, this study demonstrates that for postoperative cardiac surgery patients, bladder temperature measurement performs equal or better than rectal temperature measurement. Diuresis did not interfere with bladder temperature measurement.

We believe that both from the patient's perspective and from the nursing perspective, bladder temperature measurement has sufficient advantages in terms of accuracy, patient comfort and cost to be used in place of the cumbersome rectal temperature measurements in these patients.

\section{REFERENCES}

[1] Robb, P.J. and Shahab, R. (2001) Infrared transtympanic temperature measurement and otitis media with effusion. Internal Journal Pediatric Otorhinolaryngology, 59, 195 200. doi:10.1016/S0165-5876(01)00482-7

[2] Mravinac, C.M., Dracup, K. and Clochesy, J.M. (1989) Urinary bladder and rectal temperature monitoring during clinical hypothermia. Nursing Research, 38, 73-76. doi:10.1097/00006199-198903000-00005

[3] Pompei, F. and Pompei, M. (1996) Physicians' reference 
handbook on temperature. Exergen Corporation, Boston.

[4] Bartlett, E.M. (1996) Temperature measurement: Why and how in intensive care. Intensive Critical Care Nurse, 12, 50-54. doi:10.1016/S0964-3397(96)81698-3

[5] Horrow, J.C. and Rosenberg, H. (1988) Does urinary catheter temperature reflect core temperature during cardiac surgery? Anesthesiology, 69, 986-989. doi:10.1097/00000542-198812000-00037

[6] Lefrant, J.Y., Muller, L., de La Coussaye, J.E., Benbabaali, M., Lebris, C., Zeitoun, N., et al. (2002) Temperature measurement in intensive care patients: Comparison of urinary bladder, oesophageal, rectal, axillary, and inguinal methods versus pulmonary artery core method. Intensive Care Medicine, 29, 414-418. doi:10.1007/s00134-002-1364-9

[7] Erickson, R.S. and Meyer L.T. (1994) Accuracy of infrared ear thermometry and other temperature methods in adults. American Journal of Critical Care, 3, 40-54.

[8] Giuliano, K.K., Scott, S.S., Elliot, S. and Giuliano, A.J. (1999) Temperature measurement in critically ill orally intubated adults: A comparison of pulmonary artery core, tympanic, and oral methods. Critical Care Medicine, 27, 2188-2193. doi:10.1097/00003246-199910000-00020

[9] Sato, H., Yamakage, M., Okuyama, K., Imai, Y., Iwashita, H., Ishiyama, T. and Matsukawa, T. (2008) Urinary bladder and oesophageal temperatures correlate better in patients with high rather than low urinary flow rates during non-cardiac surgery. European Journal of Anaesthesiology, 25, 805-809. doi:10.1017/S0265021508004602

[10] Langham, G.E., Maheshwari, A., Contrera, K., You, J.,
Mascha, E. and Sessler, D.I. (2009) Noninvasive temperature monitoring in postanesthesia care units. Anesthesiology, 111, 90-96. doi:10.1097/ALN.0b013e3181a864ca

[11] Lilly, J.K., Boland, J.P. and Zekan, S. (1980) Urinary bladder temperature monitoring: A new index of body core temperature. Critical Care Medicine, 8, 742-744. doi:10.1097/00003246-198012000-00010

[12] Robinson, J., Charlton, J., Seal, R., Spady, D. and Joffres, M.R. (1998) Oesophageal, rectal, axillary, tympanic and pulmonary artery temperatures during cardiac surgery. Canadian Journal Anaesthesia, 45, 317-323. doi:10.1016/S0022-3476(98)70067-8

[13] Fallis, W.M. (2002) Monitoring urinary bladder temperature in the intensive care unit: State of the science. American Journal of Critical Care, 11, 38-45.

[14] Fulbrook, P. (1993) Core temperature measurement: A comparison of rectal, axillary and pulmonary artery blood temperature. Intensive Critical Care Nurse, 9, 217-225. doi:10.1016/S0964-3397(05)80002-3

[15] Earp, J.K. and Finlayson, D.C. (1991) Relationship between urinary bladder and pulmonary artery temperatures: A preliminary study. Heart Lung, 20, 265-270.

[16] Earp, J.K. and Finlayson, D.C. (1992) Urinary bladder/ pulmonary artery temperature ratio of less than 1 and shivering in cardiac surgical patients. American Journal of Critical Care, 1, 43-52.

[17] Moorthy S.S., Winn, B.A., Jallard, M.S., Edwards, K. and Smith, N.D. (1985) Monitoring urinary bladder temperature. Heart Lung, 14, 90-93.

\author{
ABBREVIATIONS \\ CABG: Coronary artery bypass grafting; \\ ICU: Intensive care unit; \\ Tb: Bladder temperature; \\ Tpa: Pulmonary artery temperature; \\ Tr: Rectal temperature; \\ PAC: Pulmonary artery catheter; \\ SD: Standard deviation.
}

